\title{
Nomogram For Preoperative Prediction Of Microvascular Invasion Risk In Hepatocellular Carcinoma
}

This article was published in the following Dove Press journal: Cancer Management and Research

\author{
Guangtong Deng' \\ Lei Yao' \\ Furong Zeng ${ }^{2}$ \\ Liang Xiao',* \\ Zhiming Wang $\mathbb{D}^{1, *}$
}

'General Surgery Department, Xiangya Hospital, Central South University, Changsha, People's Republic of China;

${ }^{2}$ Xiangya School of Medicine, Central South University, Changsha, People's Republic of China

*These authors contributed equally to this work

Correspondence: Zhiming Wang; Liang

Xiao

General Surgery Department, Xiangya

Hospital, Central South University,

Xiangya Road 87, Changsha 410008,

People's Republic of China

Tel +86/8773132682

Email a846957007@I63.com;

xiaoliangrick@csu.edu.cn
Objective: To preoperatively predict the microvascular invasion (MVI) risk in hepatocellular carcinoma (HCC) using nomogram.

Methods: A retrospective cohort of 513 patients with HCC hospitalized at Xiangya Hospital between January 2014 and December 2018 was included in the study. Univariate and multivariate analysis was performed to identify the independent risk factors for MVI. Based on the independent risk factors, nomogram was established to preoperatively predict the MVI risk in HCC. The accuracy of nomogram was evaluated by using receiver operating characteristic (ROC) curve, calibration curve and decision curve analysis (DCA).

Results: Tumor size $(\mathrm{OR}=1.17,95 \%$ CI: $1.11-1.23, \mathrm{p}<0.001)$, preoperative AFP level greater than $155 \mathrm{ng} / \mathrm{mL}(\mathrm{OR}=1.65,95 \% \mathrm{CI}: 1.13-2.39, \mathrm{p}=0.008)$ and $\mathrm{NLR}(\mathrm{OR}=1.14,95 \% \mathrm{CI}: 1.00-1.29$, $\mathrm{p}=0.042$ ) were the independent risk factors for MVI. Incorporating these 3 factors, nomogram was established with the concordance index of 0.71 (95\% CI, 0.66-0.75) and well-fitted calibration curves. DCA confirmed that using this nomogram added more benefit compared with the measures that treat all patients or treat none patients. At the cutoff value of predicted probability $\geq 0.44$, the model demonstrated sensitivity of $61.64 \%$, specificity of $71.53 \%$, positive predictive value (PPV) of $64.13 \%$, and negative predictive value (NPV) of $69.31 \%$.

Conclusion: Nomogram was established for preoperative prediction of the MVI risk in HCC patients, and better therapeutic choice will be made if it was applied in clinical practice.

Keywords: microvascular invasion, MVI, hepatocellular carcinoma, HCC, preoperative prediction, independent risk factors, nomogram

\section{Introduction}

Hepatocellular carcinoma (HCC) accounts for $75 \%$ to $85 \%$ of primary liver cancer, which is the sixth most common cancer and the fourth leading cause of cancerrelated mortality worldwide. ${ }^{1}$ Nowadays, surgical resection and liver transplantation remain the mainstay of curative approach for HCC. However, the 5-year recurrence rate is as high as $70 \%$ after surgical resection and even $25 \%$ after transplantation. ${ }^{2,3}$

Microvascular invasion (MVI) is defined as the presence of cancer cell clusters in the branch of portal vein under the microscopy. ${ }^{4}$ MVI is an extremely important independent risk factor of postoperative HCC recurrence after curative therapy. ${ }^{5-9}$ MVI can not only help clinicians to develop therapeutic schedules after surgery, but also guide surgeons on whether to perform liver transplantation beyond Milan Criteria, 
anatomical liver resection and widening of the surgical margin. ${ }^{10-13}$ However, in current clinical practice, the diagnosis of MVI still depends on the pathological examination after liver resection or transplantation. ${ }^{4}$ Therefore, an accurate preoperative prediction is of great importance for clinical decision-making in choosing the best strategy to manage the individual HCC patient.

Many efforts on preoperative prediction of MVI have been made in recent years. Radiomic analysis of contrastenhanced CT and gadoxetic acid-enhanced MRI was applied for the prediction of MVI ${ }^{14-16}$ besides, some teams evaluated the risk of MVI based on the ultrasound-related radiomics score. ${ }^{17,18}$ However, all of these models relied too heavily on imaging data and did not include inflammatory indices. Inflammatory indices are reflective of the systematic inflammation which play an essential role in cancer development and progression. ${ }^{19-21}$ Also, clinical studies showed that inflammatory indices can be used to predict the prognosis and the presence of MVI. ${ }^{22-27}$ Therefore, it is necessary to combine inflammatory indices into the predictive models. The objective of our study was to develop nomograms based on imaging data as well as serum inflammatory data for MVI preoperative prediction in HCC.

\section{Methods}

\section{Patients}

With the approval of the Xiangya Hospital of Central South University, we retrospectively collected the data of HCC patients who underwent partial hepatectomy between January 2014 and December 2018. The study was conducted in compliance with the Declaration of Helsinki and relevant policies in China. Written informed consent was obtained from all patients for their data to be used for research. Patients did not receive financial compensation. The inclusion criteria were as follows: (1) patients were above 18 years; (2) underwent surgical resection; (3) pathological diagnosis of HCC with or without MVI. MVI was diagnosed as the presence of cancer cell clusters in the branch of portal vein under the microscopy. Diagnostic criteria were based on Guidelines for Diagnosis and Treatment of Primary Liver Cancer in China (2017 Edition) ${ }^{4}$ (4) imaging data and serum inflammatory data were obtained before surgery. The exclusion criteria were as follows: (1) patients were less than 18 years; (2) patients were diagnosed as metastatic tumor before; (3) unclear diagnosis of HCC with MVI or not; (4) imaging data and serum inflammatory data were unavailable.

\section{Clinicopathologic Variables}

Patients' demographic variables, including age, sex, body mass index, history of diabetes, hypertension, hepatitis $\mathrm{B}$, and hepatitis $\mathrm{C}$ were obtained based on discharge diagnosis. Patients' imaging data from contrast-enhanced MRI, contrast-enhanced CT and ultrasound were also reviewed. The following parameters were recorded: number of tumor nodules, tumor size, hypersplenotrophy, ascites and cirrhosis. Serum examination included indocyanine green retention rate at 15 mins (ICG-R15), serum $\alpha$-fetoprotein level (AFP), carcinoembryonic antigen (CEA), cancer antigen 199 (CA199), hepatitis be antigen (HBeAg), albumin (ALB), total bilirubin (TBIL), direct bilirubin (DBIL), alanine transaminase (ALT), aspartate transaminase (AST), alkaline phosphatase (ALP), prothrombin time (PT), prothrombin activity (PTA), international normalized ratio (INR), neutrophil, lymphocyte, monocyte, platelet, hemoglobin, neutrophil-to-lymphocyte ratio (NLR), platelet-tolymphocyte ratio (PLR), lymphocyte-to-monocyte ratio (LMR), AST-to-platelet ratio index (APRI) and AST-toneutrophil ratio index (ANRI). In this study, NLR was calculated by the neutrophils count divided by lymphocytes count; PLR was calculated by the platelets count divided by lymphocytes count; LMR was calculated by the lymphocytes count divided by monocytes count. APRI was obtained using the following formula: APRI $=[$ AST level $(/ \mathrm{ULN}) /$ Platelet counts $\left.\left(10^{9} / \mathrm{L}\right)\right] \times 100$. ANRI was measured by AST divided by neutrophil count.

\section{Statistical Analysis}

Receiver operating characteristic curve analysis was used to calculate the optimal cutoff values based on the maximum of the Youden index. Continuous variables were expressed as mean $\pm \mathrm{SD}$ and compared using the Student's $t$-test. Categorical variables were expressed as frequency and compared using the chi-square test or Fisher exact test. Factors were subjected to multivariate logistic regression analysis to identify the independent MVI predictors if their $P$ values were less than 0.05 in the univariate analysis. According to the independent MVI predictors, a nomogram was formulated by using EmpowerStats software and the rms package of R, version 3.0 (http://www.r-project.org/). ${ }^{28,29}$ Concordance index ( $\mathrm{C}$ index) was employed to quantify the discrimination of the nomogram and a calibration curve with 1000 bootstrap samples was employed to measure the accuracy of the nomogram. The decision curve analysis (DCA) was conducted to evaluate the clinical utility of the nomogram through 
quantifying net benefits against a range of threshold probabilities. All analyses were performed using SPSS 22.0 (SPSS Inc., Chicago, IL, USA), EmpowerStats, State SE and R 3.1.2 software (Institute for Statistics andMathematics, Vienna, Austria).

\section{Results}

\section{Demographic Characteristics}

A total of $513 \mathrm{HCC}$ patients were enrolled in the present study, including 449 males and 64 females. The average age was $52.02 \pm 11.5$ years old. Two hundred and thirty-two (45.2\%) patients were complicated with MVI according to histopathological reports. About $84.2 \%$ of HCC patients was accompanied with hepatitis $\mathrm{B}$ virus infection, $2.3 \%$ with hepatitis $C$ virus infection and $13.5 \%$ had no evidence of hepatitis. About $17.7 \%$ of patients suffered from hypertension and 7.4\% suffered from diabetes. Univariate analysis showed that patients with MVI shared similar demographic characteristics to patients without MVI (Table 1).

\section{Univariate And Multivariate Analysis Of MVI-Related Factors}

In consideration of the significant variation of AFP value, we plotted the receiver operating characteristic (ROC) curve to determine the AFP cutoff value (Figure 1). Thus, the patients were dichotomized into groups of "high AFP ( $\geq 155 \mathrm{ng} / \mathrm{mL})$ " and "low AFP $(<155 \mathrm{ng} / \mathrm{mL})$ ". In the univariate analysis, tumor size, TBIL ( $p=0.048)$, DBIL $(p=0.037)$, AST $(p=0.001)$, PLR $(p=0.002), \quad A F P \geq 155 n g / m L \quad(p<0.001), \quad$ lymphocytes $(p=0.015)$, NLR $(p<0.001)$ and LMR $(p=0.003)$ were potential risk factors for MVI (Table 1). Subsequently, all these potential risk factors were recruited into multivariate analysis to adjust the effects of covariates for the presence of MVI. Only tumor size $(\mathrm{OR}=1.17,95 \% \mathrm{CI}$ : $1.11-1.23, \quad \mathrm{p}<0.001), \quad \mathrm{AFP} \geq 155 \mathrm{ng} / \mathrm{mL} \quad(\mathrm{OR}=1.65,95 \%$ CI: $1.13-2.39, \mathrm{p}=0.008)$ and NLR $(\mathrm{OR}=1.14,95 \% \mathrm{CI}$ : $1.00-1.29, \mathrm{p}=0.042$ ) were the independent risks for MVI (Figure 2).

\section{Development And Validation Of An MVI- Predicting Nomogram}

The independent risk factors for MVI were further employed to establish the MVI risk estimation nomogram (Figure 3). The nomogram was internally validated using the $\mathrm{C}$ index and the bootstrap validation method. The nomogram showed a good discrimination for predicting the risk of MVI, with an $\mathrm{C}$ index of 0.71 (95\% CI, 0.66-0.75) (Figure 4). In addition, calibration plots graphically showed good agreement between prediction and actual histopathologic confirmation on surgical specimens (Figure 5). DCA showed that using this nomogram to predict MVI added more benefit compared with the measures that treat all patients or treat none patients (Figure 6).

\section{Risk Of MVI Based On The Nomogram Scores}

Sensitivity and specificity for predicting MVI at different cutoff values are summarized in Table 2. Although higher cutoff values resulted in higher specificity, sensitivity rapidly dropped to a point at which the model may omit many true MVI patients. According to the maximum of the Youden index, the optimal cutoff values for the MVI-predicting nomogram predicted probability were set to be 0.44 . The sensitivity, specificity, positive predictive value, and negative predictive value, when used in differentiating the presence from absence of MVI, were $61.64 \%, 71.53 \%, 64.13 \%$, and $69.31 \%$, respectively (Table 3 ).

\section{Discussion}

MVI was usually regarded as an important prognostic factor for HCC after curative treatment. However, recent studies show that MVI could help with clinical decisionmaking before surgery. For example, in 2009, Mazzaferro $\mathrm{V}$ showed that there was no significant difference in the 5-year survival rate after liver transplantation between using Milan criteria and MVI-negative Up-to-seven criteria. ${ }^{11}$ Besides, in 2017, Zhao and his team found that patients with MVI benefited from anatomical hepatectomy in terms of disease-free survival rate compared with non-anatomical hepatectomy. ${ }^{13}$ In 2019, a multi-center retrospective study showed that MVI-positive patients with widened surgical margin had longer disease-free survival and overall survival. ${ }^{10}$ Therefore, it is meaningful to predict the MVI preoperatively considering the importance of MVI for clinical decision-making.

In our analysis, we found that tumor size, AFP and NLR were the independent risk factors for MVI in HCC. Based on the risk factors, we established the nomogram to predict the presence of MVI. As for tumor size, Pawlik found it to be positively correlated 
Table I Characteristics Of Patients Compared On The Basis Of Tumor Microvascular Invasion (MVI)

\begin{tabular}{|c|c|c|c|c|}
\hline Variables & All Patients $(n=5 \mid 3)$ & MVI-Positive $(n=232)$ & MVI-Negative $(n=28 I)$ & P-Value \\
\hline \multicolumn{5}{|c|}{ Demographics and history } \\
\hline Age (years) & $52.02 \pm|1.5|$ & $51.18 \pm 11.80$ & $52.7| \pm| 1.24$ & 0.133 \\
\hline Sex & & & & \\
\hline Man & 449 & 206 & 243 & 0.502 \\
\hline Woman & 64 & 26 & 38 & \\
\hline BMI & $22.93 \pm 3.08$ & $22.67 \pm 3.10$ & $23.15 \pm 3.06$ & 0.159 \\
\hline \multicolumn{5}{|l|}{ Diabetes } \\
\hline Yes & 38 & 12 & 26 & 0.091 \\
\hline No & 475 & 220 & 255 & \\
\hline \multicolumn{5}{|l|}{ Hypertension } \\
\hline Yes & 91 & 39 & 52 & 0.644 \\
\hline No & 422 & 193 & 229 & \\
\hline \multicolumn{5}{|l|}{ Etiology } \\
\hline HBV & 432 & 201 & 231 & 0.237 \\
\hline $\mathrm{HCV}$ & 12 & 3 & 9 & \\
\hline Others & 69 & 28 & 41 & \\
\hline \multicolumn{5}{|l|}{ Preoperative blood tests } \\
\hline ICG-RI5 (\%) & $6.79 \pm 7.14$ & $7.50 \pm 8.78$ & $6.20 \pm 5.35$ & 0.141 \\
\hline \multicolumn{5}{|l|}{$\operatorname{AFP}(\mathrm{ng} / \mathrm{mL})$} \\
\hline$\leq 155$ & 250 & 92 & 158 & $<0.001$ \\
\hline$>155$ & 263 & 140 & 123 & \\
\hline CEA (ng/mL) & $2.73 \pm 3.77$ & $2.92 \pm 5.15$ & $2.57 \pm 1.94$ & 0.315 \\
\hline CAI99 (ng/mL) & $26.47 \pm 27.52$ & $26.85 \pm 25.99$ & $26.17 \pm 28.70$ & 0.809 \\
\hline \multicolumn{5}{|l|}{$\mathrm{HBeAg}$} \\
\hline Yes & 321 & 154 & 167 & 0.136 \\
\hline No & 181 & 74 & 107 & \\
\hline $\operatorname{ALB}(g / L)$ & $40.90 \pm 4.55$ & $40.93 \pm 4.42$ & $40.88 \pm 4.65$ & 0.897 \\
\hline TBIL $(\mu \mathrm{mol} / \mathrm{L})$ & $14.49 \pm 10.48$ & $15.50 \pm 13.04$ & $13.66 \pm 7.69$ & 0.048 \\
\hline $\mathrm{DBIL}(\mu \mathrm{mol} / \mathrm{L})$ & $6.58 \pm 6.47$ & $7.24 \pm 7.87$ & $6.04 \pm 4.98$ & 0.037 \\
\hline ALT (U/L) & $43.31 \pm 40.05$ & $45.40 \pm 48.74$ & $41.58 \pm 31.09$ & 0.282 \\
\hline AST (U/L) & $50.93 \pm 46.01$ & $58.4 I \pm 56.8 I$ & $44.75 \pm 33.52$ & 0.001 \\
\hline ALP (U/L) & $116.89 \pm 60.12$ & $118.86 \pm 52.92$ & II $5.53 \pm 65.50$ & 0.792 \\
\hline PT (s) & $13.86 \pm 5.38$ & $|3.64 \pm| .28$ & $14.05 \pm 7.18$ & 0.383 \\
\hline PTA (\%) & $96.92 \pm 14.15$ & $96.47 \pm 14.27$ & $97.30 \pm 14.07$ & 0.509 \\
\hline INR & $1.07 \pm 0.10$ & $1.07 \pm 0.10$ & $1.07 \pm 0.10$ & 0.612 \\
\hline Neutrophil $\left(10^{9} / \mathrm{L}\right)$ & $3.43 \pm 2.11$ & $3.56 \pm 1.52$ & $3.33 \pm 2.49$ & 0.219 \\
\hline Lymphocyte $\left(10^{9} / \mathrm{L}\right)$ & $1.52 \pm 0.99$ & $I .4 I \pm 0.56$ & $1.63 \pm 1.22$ & 0.015 \\
\hline Monocyte $\left(10^{9} / \mathrm{L}\right)$ & $0.82 \pm|.7|$ & $0.75 \pm 1.52$ & $0.88 \pm 1.86$ & 0.380 \\
\hline Platelet $\left(10^{9} / \mathrm{L}\right)$ & $158.10 \pm 74.27$ & $164.53 \pm 69.11$ & $152.79 \pm 78.00$ & 0.075 \\
\hline $\mathrm{HB}(g / L)$ & $|42.44 \pm 6| .34$ & $|4| .2| \pm| 9.8 \mid$ & $|43.47 \pm 8| .02$ & 0.679 \\
\hline NLR & $2.61 \pm 1.74$ & $2.94 \pm 2.05$ & $2.34 \pm 1.37$ & $<0.001$ \\
\hline PLR & II $8.36 \pm 69.43$ & $128.89 \pm 64.17$ & $109.65 \pm 72.45$ & 0.002 \\
\hline LMR & $3.36 \pm 2.17$ & $3.05 \pm 1.43$ & $3.62 \pm 2.60$ & 0.003 \\
\hline APRI & $1.35 \pm 5.56$ & $1.17 \pm 1.04$ & $1.50 \pm 7.45$ & 0.499 \\
\hline ANRI & $18.00 \pm 18.54$ & $18.86 \pm 18.99$ & $17.29 \pm 18.16$ & $0.34 I$ \\
\hline $\begin{array}{l}\text { Preoperative imaging } \\
\text { Tumor number }\end{array}$ & & & & \\
\hline
\end{tabular}

(Continued) 
Table I (Continued).

\begin{tabular}{|l|l|l|l|l|}
\hline Variables & All Patients (n=5 I3) & MVI-Positive (n=232) & MVI-Negative (n=28I) & P-Value \\
\hline $\begin{array}{l}\text { Solitary } \\
\text { Multiple }\end{array}$ & 442 & 200 & 242 & 1.000 \\
Tumor size (cm) & $7 I$ & 32 & 39 & $<.19 \pm 3.25$ \\
\hline $\begin{array}{l}\text { Splenomegaly } \\
\text { Yes }\end{array}$ & $6.29 \pm 3.91$ & $7.62 \pm 4.21$ & & 41 \\
No & 76 & 35 & 240 & 0.901 \\
\hline $\begin{array}{l}\text { Ascites } \\
\text { Yes }\end{array}$ & 437 & 197 & & 8 \\
No & 17 & 9 & 273 & 0.622 \\
\hline $\begin{array}{l}\text { Liver cirrhosis } \\
\text { Yes }\end{array}$ & 496 & 223 & & 166 \\
No & 305 & 139 & 115 & 0.857 \\
\hline
\end{tabular}

Notes: Categorical variables are expressed as frequency. Continuous variables are expressed as mean (standard deviation).

Abbreviations: ICG-RI5, indocyanine green retention rate at $15 \mathrm{~min}$; AFP, $\alpha$-fetoprotein level; CEA, carcinoembryonic antigen; CAI99, cancer antigen I99; HBeAg, hepatitis be antigen; ALB, albumin; TBIL, total bilirubin; DBIL, direct bilirubin; ALT, alanine transaminase; AST, aspartate transaminase; ALP, alkaline phosphatase; PT, prothrombin time; PTA, prothrombin activity; INR, international normalized ratio; NLR, neutrophil-to-lymphocyte ratio; PLR, platelet-to-lymphocyte ratio; LMR, lymphocyte-to-monocyte ratio; APRI, AST-to-platelet ratio index; ANRI, AST-to-neutrophil ratio index.

with the MVI risk. For the tumor size less than $3 \mathrm{~cm}$, the MVI risk was about 25\%; for the tumor size between $3 \mathrm{~cm}$ and $5 \mathrm{~cm}$, the MVI risk was about $40 \%$; for the tumor size between $5 \mathrm{~cm}$ and $6.5 \mathrm{~cm}$, the MVI risk moved up to $63 \%$. Interestingly, tumor size above $5 \mathrm{~cm}$ is an independent risk factor for MVI in HCC. ${ }^{30}$ Now tumor size was widely accepted as a risk factor for MVI while the tumor size cut-off value for

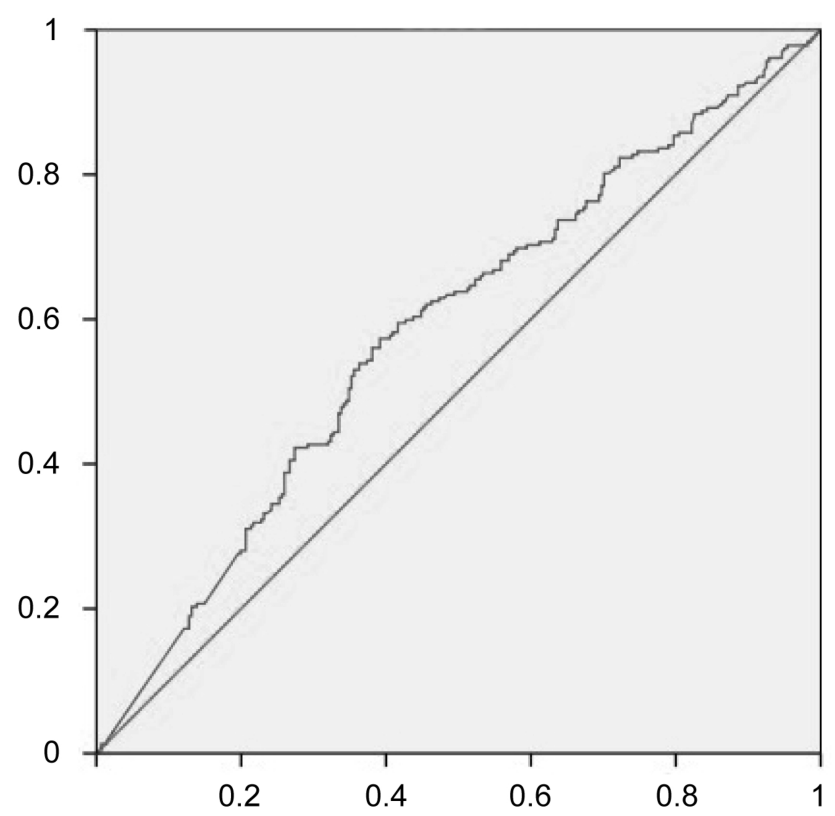

Figure I Receiver operating characteristic (ROC) curves for AFP in HCC patients according to microvascular invasion (MVI)-positive. predicting MVI is still controversial. ${ }^{31-39}$ Our study analyzed tumor size as a continuous variable, which can keep more information compared to other studies regarding tumor size as a discontinuous variable. As for AFP, due to the significant variation, we turned it into a discontinuous variable based on the maximum of Youden index. Back to 2005, Pawlik suggested that AFP above $1000 \mathrm{ng} / \mathrm{mL}$ is an independent risk factor for poor prognosis of HCC patients. $^{30}$ In 2010, Cucchetti demonstrated that AFP is an independent risk factor for MVI in HCC patients. ${ }^{40}$ However, Hirokawa arrived at a different conclusion which suggested that AFP was not related to MVI. ${ }^{41}$ Our analysis showed that AFP could be applied to predict the risk of MVI. As for NLR, Zheng showed that there was a significant difference between MVI-positive and MVInegative group in NLR level using univariate analysis, but no difference was observed after multivariate analysis. ${ }^{19}$ Li's study also conformed this point. $^{24}$ However, $\mathrm{Yu}$ and his team had a different opinion that NLR was a useful biomarker for predicting MVI in

\begin{tabular}{|c|c|c|c|}
\hline Independent risk factors & Forest plot & OR $(95 \% \mathrm{Cl})$ & $\mathrm{P}$ \\
\hline AFP (>155 vs. $\leq 155 \mathrm{ng} / \mathrm{ml})$ & - & $1.65(1.13,2.39)$ & 0.008 \\
\hline NLR & $\rightarrow$ & $1.14(1.00,1.29)$ & 0.042 \\
\hline Tumor size $(\mathrm{cm})$ & $\rightarrow$ & $1.17(1.11,1.23)$ & $<0.001$ \\
\hline
\end{tabular}

Figure 2 Plot of independent risk factors predicting MVI based on multivariate logistic regression analysis. 


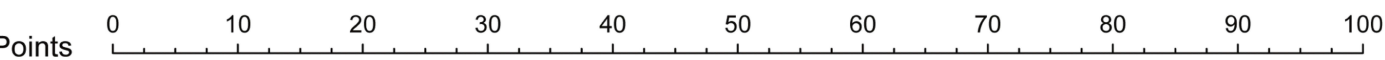
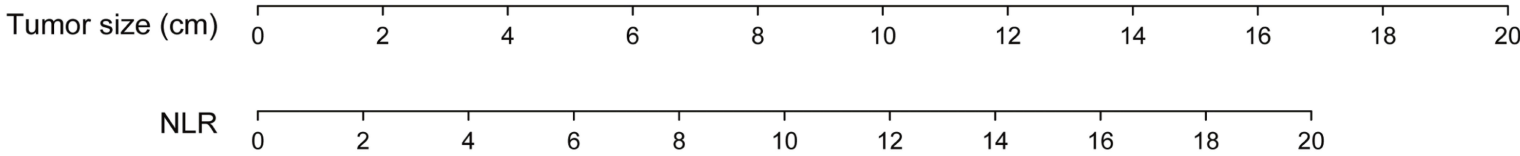
$>155$

$\operatorname{AFP}(\mathrm{ug} / \mathrm{l}) \underset{\leq 155}{ }$

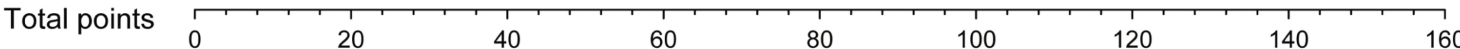

Probability of MVI presence

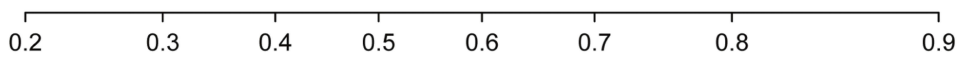

Figure 3 Nomogram to predict the risk of MVI preoperatively in HCC.

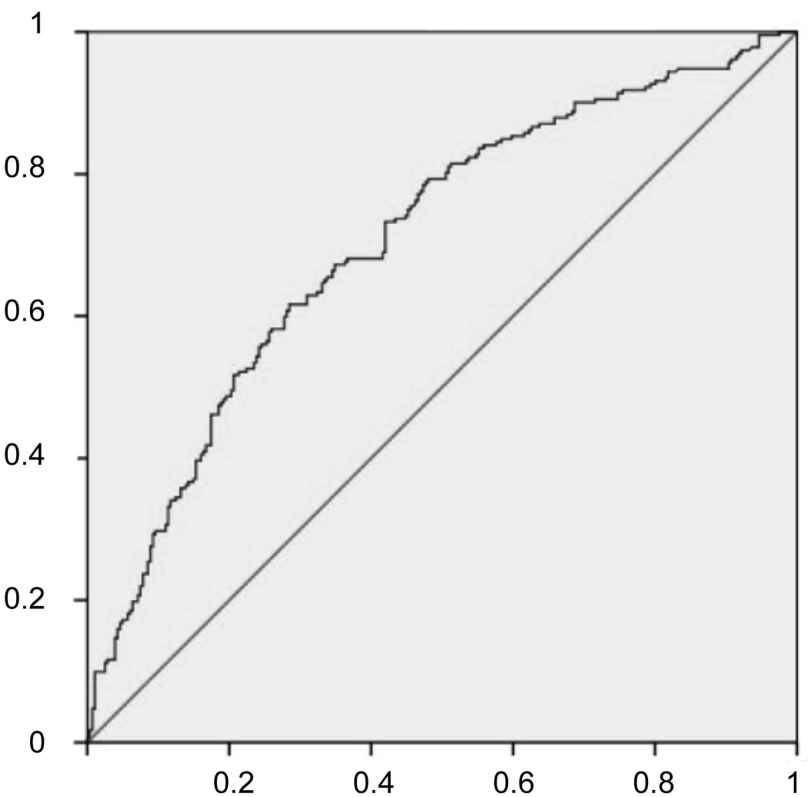

Figure 4 The accuracy of the nomogram for predicting MVI using ROC curve.

patients with $\mathrm{HCC}^{42}$ In a word, our analysis highlights the predictive value of tumor size, AFP and NLR based on the data from Xiangya hospital.

For the clinical use of the nomogram, we summarized the sensitivity, specificity, positive predictive value, and negative predictive value in estimating the risk of MVI at different cutoff values. Besides, we determined the optimal cutoff values of predicted probability for MVI to be 0.44 based on the maximum of the Youden index. This means HCC patients with a predicted probability of 0.44 or less are a low-risk subgroup of MVI. Based on these preoperative predic- tions, a low-risk subgroup of MVI can still receive liver transplantation if they do not meet Milan criteria but meet Up-to-seven criteria due to no difference in the 5-year survival rate. ${ }^{11}$ Besides, the preoperative prediction for MVI could guide surgical management in the selection of operation methods (anatomical or non-anatomical resection) and width of surgical margins. Furthermore, it may serve as a selection tool during randomized clinical trials for evaluating the efficacy of liver resection in HCC patients with different MVI risks.

To our knowledge, this is the first nomogram of combining NLR into predictive models of MVI in HCC. However, our study had some limitations. First, this analysis was based on data from a single hospital. Second, this study is a retrospective study and some markers such as DCP and AFP-LC3 which are regarded as the independent risk factors for MVI are not included in our analysis due to limited data availability. Third, an external validation is necessary to confirm the prediction value of the nomogram. Finally, due to analysis based on clinicopathologic data, specific markers to estimate MVI might further improve the accuracy of the nomogram.

In conclusion, we demonstrated that tumor size, AFP and NLR are the independent risk factors of MVI in HCC. Through combining the independent risk factors, we have established a nomogram. The model could optimally estimate the risk of MVI in HCC patients and help with clinical decision-making before surgery. 


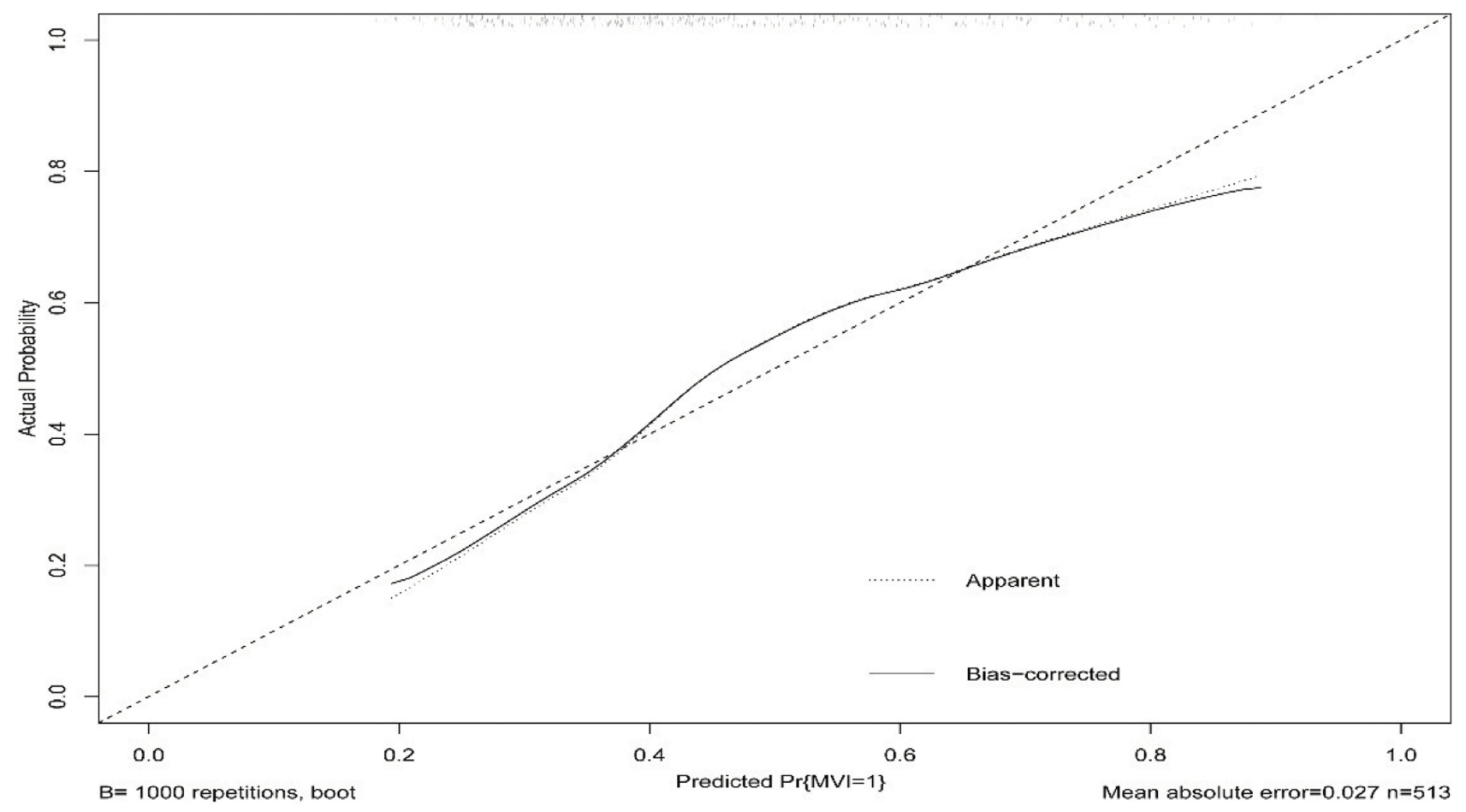

Figure 5 Calibration plot of the nomogram for predicting the risk of MVI.

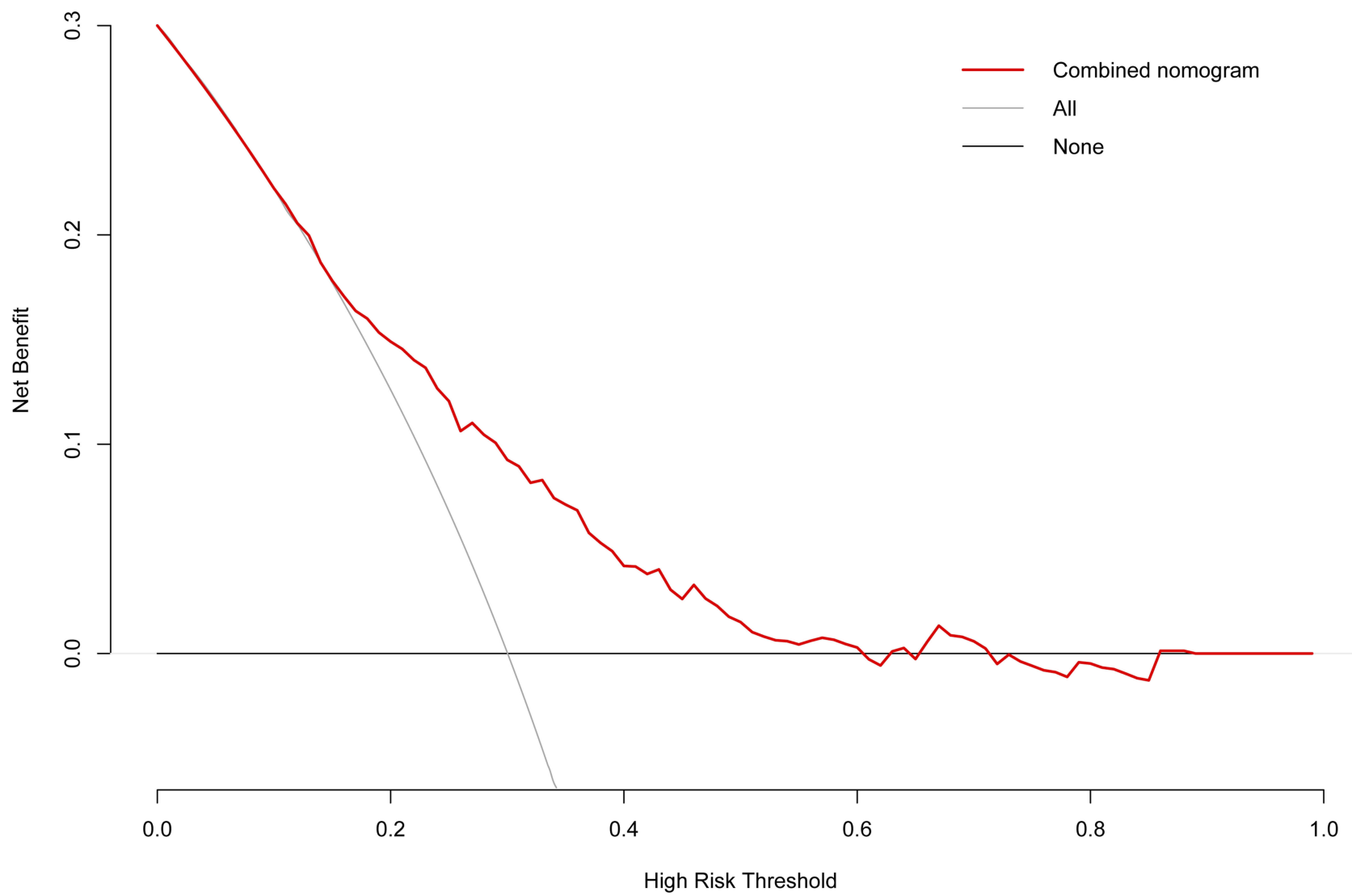

Figure 6 Decision curve analysis of our nomogram. 
Table 2 Accuracy Of The Nomogram For Estimating The Risk Of MVI At Different Cutoff Values

\begin{tabular}{|c|c|c|c|c|c|}
\hline Predicted Probability & Threshold & Sensitivity & Specificity & PPV & NPV \\
\hline 0.20 & -1.35 & $100 \%$ & $2 \%$ & $46 \%$ & $100 \%$ \\
\hline 0.30 & -0.85 & $90 \%$ & $28 \%$ & $51 \%$ & $77 \%$ \\
\hline 0.40 & -0.40 & $68 \%$ & $58 \%$ & $57 \%$ & $69 \%$ \\
\hline 0.50 & 0 & $50 \%$ & $80 \%$ & $67 \%$ & $66 \%$ \\
\hline 0.60 & 0.41 & $32 \%$ & $89 \%$ & $70 \%$ & $61 \%$ \\
\hline 0.70 & 0.85 & $18 \%$ & $94 \%$ & $72 \%$ & $58 \%$ \\
\hline 0.80 & 1.39 & $9 \%$ & $99 \%$ & $87 \%$ & $57 \%$ \\
\hline
\end{tabular}

Abbreviations: NPV, negative predictive value; PPV, positive predictive value.

Table 3 Accuracy Of The Nomogram For Estimating The Risk Of MVI At Optimal Threshold Value

\begin{tabular}{|l|l|}
\hline Variables & Value \\
\hline Sensitivity & $61.64 \%$ \\
Specificity & $71.53 \%$ \\
Positive predictive value & $64.13 \%$ \\
Negative predictive value & $69.31 \%$ \\
Positive likelihood ratio & 2.17 \\
Negative likelihood ratio & 0.54 \\
ROC area (95\% Cl) & $0.71(0.66-0.75)$ \\
Optimal threshold & -0.25 \\
Predicted probability & 0.44 \\
\hline
\end{tabular}

Abbreviations: $\mathrm{ROC}$, receiver operating characteristic; $\mathrm{Cl}$, confidence intervals.

\section{Funding}

This work was supported by the grants from the National Natural Science Foundation of China (No. 81300342 http://www.nsfc.gov.cn).

\section{Disclosure}

The authors report no conflicts of interest in this work.

\section{References}

1. Bray F, Ferlay J, Soerjomataram I, Siegel RL, Torre LA, Jemal A. Global cancer statistics 2018: GLOBOCAN estimates of incidence and mortality worldwide for 36 cancers in 185 countries. CA Cancer $J$ Clin. 2018;68(6):394-424. doi:10.3322/caac.21492

2. Akoad ME, Pomfret EA. Surgical resection and liver transplantation for hepatocellular carcinoma. Clin Liver Dis. 2015;19(2):381-399. doi:10.1016/j.cld.2015.01.007

3. Bruix J, Gores GJ, Mazzaferro V. Hepatocellular carcinoma: clinical frontiers and perspectives. Gut. 2014;63(5):844-855. doi:10.1136/ gutjnl-2013-306627

4. Zhou J, Sun HC, Wang Z, et al. Guidelines for diagnosis and treatment of primary liver cancer in China (2017 Edition). Liver Cancer. 2018;7 (3):235-260. doi:10.1159/000488035

5. Jonas S, Bechstein WO, Steinmuller T, et al. Vascular invasion and histopathologic grading determine outcome after liver transplantation for hepatocellular carcinoma in cirrhosis. Hepatology. 2001;33(5):1080-1086. doi:10.1053/jhep.2001.23561
6. Huang Y, Zhang Z, Zhou Y, et al. Should we apply sorafenib in hepatocellular carcinoma patients with microvascular invasion after curative hepatectomy[J]. OncoTargets and Therapy. 2019;541-548.

7. Pote N, Cauchy F, Albuquerque M, et al. Performance of PIVKA-II for early hepatocellular carcinoma diagnosis and prediction of microvascular invasion. J Hepatol. 2015;62(4):848-854. doi:10.1016/j.jhep.2014. 11.005

8. Roayaie S, Blume IN, Thung SN, et al. A system of classifying microvascular invasion to predict outcome after resection in patients with hepatocellular carcinoma. Gastroenterology. 2009;137(3):850855. doi:10.1053/j.gastro.2009.06.003

9. Rodriguez-Peralvarez M, Luong TV, Andreana L, Meyer T, Dhillon AP, Burroughs AK. A systematic review of microvascular invasion in hepatocellular carcinoma: diagnostic and prognostic variability. Ann Surg Oncol. 2013;20(1):325-339. doi:10.1245/s10434-012-2513-1

10. Soonthornchai W, Tangtanatakul P, Meephansan J, et al. Down-regulation of miR-155 after treatment with narrow-band UVB and methotrexate associates with apoptosis of keratinocytes in psoriasis. Asian Pac J Allergy Immunol. 2019.

11. Mazzaferro V, Llovet JM, Miceli R, et al. Predicting survival after liver transplantation in patients with hepatocellular carcinoma beyond the Milan criteria: a retrospective, exploratory analysis. Lancet Oncol. 2009;10(1):35-43. doi:10.1016/S1470-2045(08)70284-5

12. Ye JZ, Chen JZ, Li ZH, et al. Efficacy of postoperative adjuvant transcatheter arterial chemoembolization in hepatocellular carcinoma patients with microvascular invasion. World J Gastroenterol. 2017;23 (41):7415-7424. doi:10.3748/wjg.v23.i41.7415

13. Zhao H, Chen $\mathrm{C}$, Gu S, et al. Anatomical versus non-anatomical resection for solitary hepatocellular carcinoma without macroscopic vascular invasion: a propensity score matching analysis. J Gastroenterol Hepatol. 2017;32(4):870-878. doi:10.1111/jgh.13603

14. Lee S, Kim SH, Lee JE, Sinn DH, Park CK. Preoperative gadoxetic acid-enhanced MRI for predicting microvascular invasion in patients with single hepatocellular carcinoma. J Hepatol. 2017;67(3):526534. doi:10.1016/j.jhep.2017.04.024

15. Wei Z, Wenguang L, Huaping L, et al. Preoperative prediction of microvascular invasion of hepatocellular carcinoma with IVIM diffusion-weighted MR imaging and Gd-EOB-DTPA-enhanced MR imaging[J]. PLOS ONE. 2018; 13(5):e0197488.

16. Banerjee S, Wang DS, Kim HJ, et al. A computed tomography radiogenomic biomarker predicts microvascular invasion and clinical outcomes in hepatocellular carcinoma. Hepatology. 2015;62(3):792800. doi: $10.1002 /$ hep. 27877

17. Hu HT, Wang Z, Huang XW, et al. Ultrasound-based radiomics score: a potential biomarker for the prediction of microvascular invasion in hepatocellular carcinoma. Eur Radiol. 2019;29(6):2890-2901. doi:10. 1007/s00330-018-5797-0

18. Wang Z, Wang W, Liu GJ, et al. The role of quantitation of real-time 3-dimensional contrast-enhanced ultrasound in detecting microvascular invasion: an in vivo study. Abdom Radiol (NY). 2016;41 (10):1973-1979. doi:10.1007/s00261-016-0804-x 
19. Zeng F, Chen B, Zeng J, et al. Preoperative neutrophil-lymphocyte ratio predicts the risk of microvascular invasion in hepatocellular carcinoma: A meta-analysis. Int J Biol Markers. 2019;34(3):213-220.

20. Honda M, Kubes P. Neutrophils and neutrophil extracellular traps in the liver and gastrointestinal system. Nat Rev Gastroenterol Hepatol. 2018;15(4):206-221. doi:10.1038/nrgastro.2017.183

21. Ringelhan M, Pfister D, O’Connor T, Pikarsky E, Heikenwalder M. The immunology of hepatocellular carcinoma. Nat Immunol. 2018;19 (3):222-232. doi:10.1038/s41590-018-0044-Z

22. Bruix J, Cheng AL, Meinhardt G, Nakajima K, De Sanctis Y, Llovet J. Prognostic factors and predictors of sorafenib benefit in patients with hepatocellular carcinoma: analysis of two phase III studies. $J$ Hepatol. 2017;67(5):999-1008. doi:10.1016/j.jhep.2017.06.026

23. Li C, Pei Q, Zhu H, et al. Survival nomograms for stage III colorectal cancer[J]. Medicine. 2018;97(49).

24. Xiong Y, Cao H, Zhang Y, et al. Nomogram-Predicted Survival of Breast Cancer Brain Metastasis: a SEER-Based Population Study[J]. World Neurosurgery, 2019.

25. Motomura T, Shirabe K, Mano Y, et al. Neutrophil-lymphocyte ratio reflects hepatocellular carcinoma recurrence after liver transplantation via inflammatory microenvironment. $J$ Hepatol. 2013;58(1):5864. doi:10.1016/j.jhep.2012.08.017

26. Rungsakulkij N, Mingphruedhi S, Suragul W, Tangtawee P, Muangkaew P, Aeesoa S. Platelet-to-lymphocyte ratio and large tumor size predict microvascular invasion after resection for hepatocellular carcinoma. Asian Pac J Cancer Prev. 2018;19(12):34353441. doi:10.31557/APJCP.2018.19.12.3435

27. Viers BR, Boorjian SA, Frank I, et al. Pretreatment neutrophil-tolymphocyte ratio is associated with advanced pathologic tumor stage and increased cancer-specific mortality among patients with urothelial carcinoma of the bladder undergoing radical cystectomy. Eur Urol. 2014;66(6):1157-1164. doi:10.1016/j.eururo.2014.02.042

28. Lei Z, Li J, Wu D, et al. Nomogram for preoperative estimation of microvascular invasion risk in hepatitis B virus-related hepatocellular carcinoma within the Milan criteria. JAMA Surg. 2016;151(4):356363. doi:10.1001/jamasurg.2015.4257

29. Pan Z, Bu Q, You H, Yang J, Liu Q, Lyu J. Determining the optimal cutoff point for lymph node density and its impact on overall survival in children with Wilms' tumor. Cancer Manag Res. 2019;11:759766. doi:10.2147/CMAR.S190138

30. Pawlik TM, Delman KA, Vauthey JN, et al. Tumor size predicts vascular invasion and histologic grade: implications for selection of surgical treatment for hepatocellular carcinoma. Liver Transpl. 2005;11(9):1086-1092. doi:10.1002/lt.20472

31. Hu HT, Wang Z, Huang XW, et al. Ultrasound-based radiomics score: a potential biomarker for the prediction of microvascular invasion in hepatocellular carcinoma. Eur Radiol. 2018;29 (6):2890-2901.
32. Hyun SH, Eo JS, Song BI, et al. Preoperative prediction of microvascular invasion of hepatocellular carcinoma using (18)F-FDG PET/ CT: a multicenter retrospective cohort study. Eur J Nucl Med Mol Imaging. 2018;45(5):720-726. doi:10.1007/s00259-017-3880-4

33. Liu M, Wang L, Zhu H, et al. a preoperative measurement of serum MicroRNA-125b may predict the presence of microvascular invasion in hepatocellular carcinomas patients. Transl Oncol. 2016;9(3):167172. doi:10.1016/j.tranon.2016.03.002

34. Min JH, Kim YK, Lim S, Jeong WK, Choi D, Lee WJ. Prediction of microvascular invasion of hepatocellular carcinomas with gadoxetic acid-enhanced MR imaging: impact of intra-tumoral fat detected on chemical-shift images. Eur J Radiol. 2015;84(6):1036-1043. doi:10.1016/j.ejrad.2015.03.002

35. Peng J, Zhang J, Zhang Q, Xu Y, Zhou J, Liu L. A radiomics nomogram for preoperative prediction of microvascular invasion risk in hepatitis B virus-related hepatocellular carcinoma. Diagn Interv Radiol. 2018;24(3):121-127. doi:10.5152/dir.2018.17467

36. Schlichtemeier SM, Pang TC, Williams NE, et al. A pre-operative clinical model to predict microvascular invasion and long-term outcome after resection of hepatocellular cancer: the Australian experience. Eur J Surg Oncol. 2016;42(10):1576-1583. doi:10.1016/j. ejso.2016.05.032

37. Ueno M, Tani M, Kawai M, et al. Triple positive tumor markers of early stage hepatocellular carcinoma is a predictor of microscopic vascular invasion and satellite nodules. HPB. 2014;16:251.

38. You Z, Chen LP, Ye H. Predictors of microvascular invasion in patients with solitary small hepatitis B related hepatocellular carcinoma. PaK J Med Sci. 2014;30(2):331-334.

39. Zhao H, Hua Y, Lu Z, et al. Prognostic value and preoperative predictors of microvascular invasion in solitary hepatocellular carcinoma $\leq 5 \mathrm{~cm}$ without macrovascular invasion. Oncotarget. 2017;8 (37):61203-61214. doi:10.18632/oncotarget.18049

40. Cucchetti A, Piscaglia F, Grigioni AD, et al. Preoperative prediction of hepatocellular carcinoma tumour grade and micro-vascular invasion by means of artificial neural network: a pilot study. $J$ Hepatol. 2010;52(6):880-888. doi:10.1016/j.jhep.2009.12.037

41. Hirokawa F, Hayashi M, Miyamoto Y, et al. Outcomes and predictors of microvascular invasion of solitary hepatocellular carcinoma. Hepatol Res. 2014;44(8):846-853. doi:10.1111/hepr.12196

42. Yu Y, Song J, Zhang R, et al. Preoperative neutrophil-to-lymphocyte ratio and tumor-related factors to predict microvascular invasion in patients with hepatocellular carcinoma. Oncotarget. 2017;8 (45):79722-79730. doi:10.18632/oncotarget.19178

\section{Publish your work in this journal}

Cancer Management and Research is an international, peer-reviewed open access journal focusing on cancer research and the optimal use of preventative and integrated treatment interventions to achieve improved outcomes, enhanced survival and quality of life for the cancer patient.
The manuscript management system is completely online and includes a very quick and fair peer-review system, which is all easy to use. Visit http://www.dovepress.com/testimonials.php to read real quotes from published authors. 